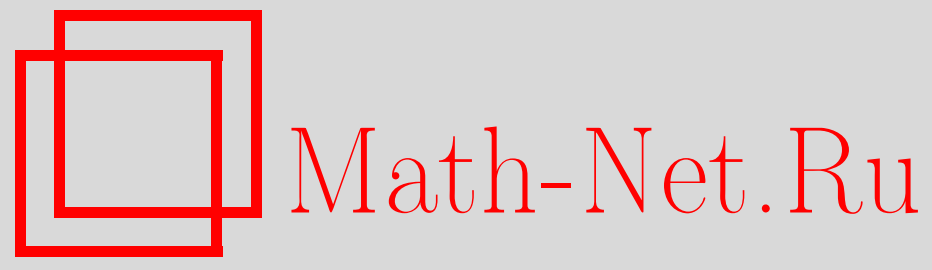

Д. А. Шляхин, Вынужденные колебания пьезокерамического цилиндра с окружной поляризацией материала, Вестн. Сам. гос. техн. ун-та. Сер. Физ.-мат. науки, 2012, выпуск 1(), 198-207

DOI: https://doi.org/10.14498/vsgtu910

Использование Общероссийского математического портала Math-Net.Ru подразумевает, что вы прочитали и согласны с пользовательским соглашением

http://www.mathnet.ru/rus/agreement

Параметры загрузки:

IP: 34.227 .88 .159

26 апреля 2023 г., 13:23:05

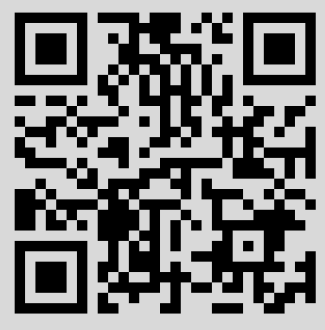




\title{
ВЫНУЖДЕННЫЕ КОЛЕБАНИЯ ПЬЕЗОКЕРАМИЧЕСКОГО ЦИЛИНДРА С ОКРУЖНОЙ ПОЛЯРИЗАЦИЕЙ МАТЕРИАЛА
}

\author{
Д. А. Шляхин \\ Самарский государственный архитектурно-строительный университет, \\ 443001, Россия, Самара, ул. Молодогвардейская, 194. \\ E-mail: d-612-mit2009@yandex.ru
}

\begin{abstract}
Рассматривается нестащионарная задача электроупругости для анизотропного пъезокерамического иилиндра конечных размеров при окружной поляризации материала в случае действия на его внешней радиальной поверхности тангенииальных напряжений и электрического потенииала, являющихся произвольными функииями аксиальной координаты и времени. Новое замкнутое решение получено методом разложения по собственным вектор-бункциям в борме структурного алгоритма конечных преобразований. Построенный алгоритм позволяет определять частоты собственных колебаний, напряженно-дебормированное состояние элемента, а такэе все компоненты индуцируемого электрического поля.
\end{abstract}

Ключевые слова: связанная задача электроупругости, иилиндр конечных размеров, осесимметричная динамическая нагрузка.

Введение. Основным элементом широкого класса импульсных преобразователей энергии является пьезокерамический цилиндр конечных размеров, работа которого основана на связанности механических и электрических полей напряжения. В случае окружной поляризации пьезоматериала данный эффект наблюдается только при распространении нестационарных волн кручения. В связи с определенной сложностью данного исследования большинство работ сводится к задачам электроупругости для бесконечного цилиндра при установившемся режиме вынужденных колебаний [1], а также исследованию нормальных осесимметричных и неосесимметричных волн кручения [2,3] в элементе конечных размеров. Также можно отметить решение, справедливое для неоднородных кристаллов тетрагональной симметрии 422 класса при действии на криволинейных поверхностях элемента динамической нагрузки в виде электрического потенциала или касательных напряжений [4].

1. Постановка задачи. В настоящей работе исследуется полый анизотропный цилиндр, занимающий в цилиндрической системе координат $\left(r_{*}, \theta, z_{*}\right)$ область $\Omega:\left\{a \leqslant r_{*} \leqslant b, 0 \leqslant \theta \leqslant 2 \pi, 0 \leqslant z_{*} \leqslant h\right\}$ и выполненный из пьезокерамического материала с наведенной окружной поляризацией.

Для рассматриваемой задачи можно сформулировать различные физически реализуемые краевые условия. Для определённости принимаем неэлектродированные торцевые плоскости свободными от механических напряжений, а радиальные поверхности полагаем электродироваными с заземлением закрепленной внутренней её части.

Краевая задача моделирует работу пьезоэлементов в приборах прямого и обратного пьезоэффекта при действии на внешней криволинейной поверхно-

Дмитрий Аверкиевич Шляхин (к.т.н., доц.), доцент, каф. сопротивления материалов и строительной механики. 
сти цилиндра соответственно тангенциальных напряжений $q^{*}\left(z_{*}, t_{*}\right)$ (вариант «а» краевых условий) и потенциала $V^{*}\left(z_{*}, t_{*}\right)$ (вариант «б»). В первом случае механическое воздействие трансформируется в электрический сигнал, при этом радиальные плоскости подключены к измерительному прибору с большим входным сопротивлением, что соответствует режиму «холостого хода» [5] (отсутствию свободных электрических зарядов), а во втором - электрическая нагрузка приводит к деформации образца.

В общем случае дифференциальные уравнения движения и электростатики однородной упругой анизотропной среды в цилиндрической системе координат записываются в виде [2]

$$
\frac{\partial \sigma_{r \theta}}{\partial r_{*}}+\frac{\partial \sigma_{z \theta}}{\partial z_{*}}+\frac{2}{r_{*}} \sigma_{r \theta}-\rho \frac{\partial^{2} \nu^{*}}{\partial t_{*}^{2}}=0, \quad \frac{\partial D_{r}}{\partial r_{*}}+\frac{D_{r}}{r_{*}}+\frac{\partial D_{z}}{\partial z_{*}}=0 .
$$

При окружной поляризации уравнения состояния пьезокерамического тела определяются следующими соотношениями $[1,2]$ :

$$
\begin{gathered}
\sigma_{r \theta}=C_{55}\left(\frac{\partial \nu^{*}}{\partial r_{*}}-\frac{\nu^{*}}{r_{*}}\right)-e_{15} E_{r}, \quad \sigma_{z \theta}=C_{55} \frac{\partial \nu^{*}}{\partial z_{*}}-e_{15} E_{z} \\
D_{r}=\varepsilon_{11} E_{r}+e_{15}\left(\frac{\partial \nu^{*}}{\partial r_{*}}-\frac{\nu^{*}}{r_{*}}\right), \quad D_{z}=\varepsilon_{11} E_{z}+e_{15} \frac{\partial \nu^{*}}{\partial z_{*}} \\
E_{z}=-\frac{\partial \phi^{*}}{\partial z_{*}}, \quad E_{r}=-\frac{\partial \phi^{*}}{\partial r_{*}}
\end{gathered}
$$

В соотношениях (1), (2) используются следующие обозначения: $t_{*}$ - время; $\sigma_{r \theta}\left(r_{*}, z_{*}, t_{*}\right), \sigma_{z \theta}\left(r_{*}, z_{*}, t_{*}\right)$ - компоненты тензора механических напряжений; $\nu^{*}\left(r_{*}, z_{*}, t_{*}\right.$ - тангенциальная составляющая вектора перемещений; $D_{r}\left(r_{*}, z_{*}, t_{*}\right), D_{z}\left(r_{*}, z_{*}, t_{*}\right), E_{r}\left(r_{*}, z_{*}, t_{*}\right), E_{z}\left(r_{*}, z_{*}, t_{*}\right), \phi^{*}\left(r_{*}, z_{*}, t_{*}\right)$ - компоненты векторов индукции, напряжённости и потенциал электрического поля; $\rho, C_{55}, e_{15}$ - объёмная плотность, модуль упругости и пьезомодуль анизотропного электроупругого материала; $\varepsilon_{11}$ - диэлектрическая проницаемость.

После подстановки (2) в (1) получаем систему дифференциальных уравнений, граничные и начальные условия рассматриваемой динамической задачи теории электроупругости в безразмерной форме:

$$
\begin{gathered}
\left(\frac{\partial^{2}}{\partial r^{2}}+\frac{1}{r} \frac{\partial}{\partial r}-\frac{1}{r^{2}}\right) \nu+\frac{\partial^{2} \nu}{\partial z^{2}}+\left(\frac{\partial^{2}}{\partial r^{2}}+\frac{2}{r} \frac{\partial}{\partial r}+\frac{\partial^{2}}{\partial z^{2}}\right) \phi-\frac{\partial^{2} \nu}{\partial t^{2}}=0 \\
\left(\frac{\partial^{2}}{\partial r^{2}}+\frac{\partial^{2}}{\partial z^{2}}\right) \nu-C_{55} \varepsilon_{11} e_{15}^{-2}\left(\frac{\partial^{2}}{\partial r^{2}}+\frac{1}{r} \frac{\partial}{\partial r}+\frac{\partial^{2}}{\partial z^{2}}\right) \phi=0 ; \\
z=0, L: \quad \sigma_{z \theta}=C_{55}\left(\frac{\partial \nu}{\partial z}+\frac{\partial \phi}{\partial z}\right)=0 \\
r=1, k: \quad D_{z}=-C_{55} \varepsilon_{11} e_{15}^{-1} \frac{\partial \phi}{\partial z}+e_{15} \frac{\partial \nu}{\partial z}=0 ; \\
\text { a) }\left.\sigma_{r \theta}\right|_{r=1}=C_{55}\left(\frac{\partial \nu}{\partial r}-\frac{\nu}{r}+\frac{\partial \phi}{\partial r}\right)=q^{*}(z, t), \\
\left.D_{r}\right|_{r=1}=-C_{55} \varepsilon_{11} e_{15}^{-1} \frac{\partial \phi}{\partial r}+e_{15}\left(\frac{\partial \nu}{\partial r}-\frac{\nu}{r}\right)=0 ; \\
\text { б) }\left.\sigma_{r \theta}\right|_{r=1}=C_{55}\left(\frac{\partial \nu}{\partial r}-\frac{\nu}{r}+\frac{\partial \phi}{\partial r}\right)=0, \quad \phi(1, z, t)=V(z, t)
\end{gathered}
$$




$$
t=0: \quad \nu(r, z, 0)=\nu_{0}(r, z), \quad \dot{\nu}(r, z, 0)=\dot{\nu}_{0}(r, z),
$$

где $\nu=\nu^{*} / b, r=r_{*} / b, z=z_{*} / b, L=h / b, k=a / b, \phi=\phi^{*} e_{15} /\left(b C_{55}\right), V=$ $=V^{*} e_{15} /\left(b C_{55}\right), t=t_{*} \sqrt{C_{55} / \rho} / b ; \nu_{0}, \dot{\nu}_{0}$ - известные в начальный момент времени тангенциальные перемещения и их скорости. Здесь и ниже точка обозначает дифференцирование по времени.

Соотношения (3)-(6) и представляют математическую формулировку рассматриваемой начально-краевой задачи электроупругости.

2. Построение общего решения. Применяем к начально-краевой задаче (3)-(6) косинус-преобразование Фурье с конечными пределами по переменной $z$. В пространстве изображений получаем следующую краевую задачу:

$$
\begin{aligned}
& \left(\frac{\partial^{2}}{\partial r^{2}}+\frac{1}{r} \frac{\partial}{\partial r}-\frac{1}{r^{2}}-j_{n}^{2}\right) \nu_{c}+\left(\frac{\partial^{2}}{\partial r^{2}}+\frac{2}{r} \frac{\partial}{\partial r}-j_{n}^{2}\right) \phi_{c}-\frac{\partial^{2} \nu_{c}}{\partial t^{2}}=0 \\
& \left(\frac{\partial^{2}}{\partial r^{2}}-j_{n}^{2}\right) \nu_{c}-C_{55} \varepsilon_{11} e_{15}^{-2}\left(\frac{\partial^{2}}{\partial r^{2}}+\frac{1}{r} \frac{\partial}{\partial r}-j_{n}^{2}\right) \phi_{c}=0 \\
& r=1, k: \quad \nu_{c}(k, n, t)=0, \quad \phi_{c}(k, n, t)=0 ; \\
& \text { a) } \quad\left[\frac{\partial \nu_{c}}{\partial r}-\nu_{c}+\frac{\partial \phi_{c}}{\partial r}\right]_{r=1}=N_{c} \\
& \quad\left[-C_{55} \varepsilon_{11} e_{15}^{-1} \frac{\partial \phi_{c}}{\partial r}+e_{15}\left(\frac{\partial \nu_{c}}{\partial r}-\nu_{c}\right)\right]_{r=1}=0 \\
& \text { б) } \quad\left[\frac{\partial \nu_{c}}{\partial r}-\nu_{c}+\frac{\partial \phi_{c}}{\partial r}\right]_{r=1}=0, \quad \phi_{c}(1, n, t)=V_{c}(n, t) ; \\
& t=0: \quad \nu_{c}(r, n, 0)=\nu_{0 c}(r, n), \quad \dot{\nu}_{c}(r, n, 0)=\dot{\nu}_{0 c}(r, n)
\end{aligned}
$$

где

$$
\begin{gathered}
\nu_{c}(r, n, t)=\int_{0}^{L} \nu(r, z, t) \cos j_{n} z d z, \quad \phi_{c}(r, n, t)=\int_{0}^{L} \phi(r, z, t) \cos j_{n} z d z, \\
\nu_{0 c}(r, n)=\int_{0}^{L} \nu_{0}(r, z) \cos j_{n} z d z, \quad \dot{\nu}_{0 c}(r, n)=\int_{0}^{L} \dot{\nu}_{0}(r, z) \cos j_{n} z d z, \\
\left.N_{c}(n, t)=\int_{0}^{L} q^{*}(z, t) C_{55}^{-1} \cos j_{n} z d z, \quad V_{c}(n, t)\right\}=\int_{0}^{L} V(z, t) \cos j_{n} z d z \\
j_{n}=n \pi / L, \quad n=0,1,2, \ldots
\end{gathered}
$$

Следует заметить, что при действии равномернораспределенной динамической нагрузки по всей радиальной поверхности цилиндра с незакрепленными и неэлектродированными торцевыми плоскостями трансформанты $N_{c}(n, t), V_{c}(n, t)$ принимают ненулевые значения только при $n=0$. В этом случае рассматривается более простая задача, связанная с анализом длинного цилиндра.

На следующем этапе решения к начально-краевой задаче (7)-(9) применяется процедура стандартизации по переменной $r$ (приведение краевых условий к однородным). Для этого трансформанты Фурье $\nu_{c}, \phi_{c}$ представляются в виде

$$
\begin{aligned}
& \nu_{c}(r, n, t)=H_{1 c}(r, n, t)+p_{c}(r, n, t), \\
& \phi_{c}(r, n, t)=H_{2 c}(r, n, t)+\chi_{c}(r, n, t),
\end{aligned}
$$


где
a) $H_{1 c}(r, n, t)=f_{1}(r) N_{c}, \quad H_{2 c}(r, n, t)=f_{2}(r) N_{c}$;
б) $H_{1 c}(r, n, t)=0$,
$H_{2 c}(r, n, t)=f_{3}(r) V_{c}$.

Подстановка (10) в (7)-(9) с учётом условий
a) $f_{1}(1)=f_{1}(k)=0$ $f_{2}(k)=0$,
$f_{1}^{\prime}(1)=C_{55} \varepsilon_{11}\left(e_{15}^{2}+C_{55} \varepsilon_{11}\right)^{-1}$,
б) $f_{3}(1)=1$,
$f_{2}^{\prime}(1)=e_{15}^{2}\left(e_{15}^{2}+C_{55} \varepsilon_{11}\right)^{-1}$;
$f_{3}(k)=f_{3}^{\prime}(1)=0$,

позволяет получить начально-краевую задачу относительно функций $p_{c}, \chi_{c}$ с однородными граничными условиями по координате $r$ :

$$
\begin{gathered}
\left(\frac{\partial^{2}}{\partial r^{2}}+\frac{1}{r} \frac{\partial}{\partial r}-\frac{1}{r^{2}}-j_{n}^{2}\right) p_{c}+\left(\frac{\partial^{2}}{\partial r^{2}}+\frac{2}{r} \frac{\partial}{\partial r}-j_{n}^{2}\right) \chi_{c}-\frac{\partial^{2} p_{c}}{\partial t^{2}}=B_{1 c} \\
\left(\frac{\partial^{2}}{\partial r^{2}}-j_{n}^{2}\right) p_{c}-C_{55} \varepsilon_{11} e_{15}^{-2}\left(\frac{\partial^{2}}{\partial r^{2}}+\frac{1}{r} \frac{\partial}{\partial r}-j_{n}^{2}\right) \chi_{c}=B_{2 c} \\
r=1, k: \quad p_{c}(k, n, t)=0, \quad \chi_{c}(k, n, t)=0 ; \\
\text { a) } \quad\left[\frac{\partial p_{c}}{\partial r}-p_{c}\right]_{r=1}=0,\left.\quad \frac{\partial \chi_{c}}{\partial r}\right|_{r=1}=0 ; \\
\text { б) } \quad\left[\frac{\partial p_{c}}{\partial r}-p_{c}+\frac{\partial \chi_{c}}{\partial r}\right]_{r=1}=0, \quad \chi_{c}(1, n, t)=0 \\
t=0: \quad p_{c}(r, n, 0)=p_{0 c}(r, n), \quad \dot{p}_{c}(r, n, 0)=\dot{p}_{0 c}(r, n)
\end{gathered}
$$

где

$$
\begin{gathered}
B_{1 c}=-\left(\frac{\partial^{2}}{\partial r^{2}}+\frac{1}{r} \frac{\partial}{\partial r}-\frac{1}{r^{2}}-j_{n}^{2}-\frac{\partial^{2}}{\partial t^{2}}\right) H_{1 c}-\left(\frac{\partial^{2}}{\partial r^{2}}+\frac{2}{r} \frac{\partial}{\partial r}-j_{n}^{2}\right) H_{2 c} \\
B_{2 c}=-\left(\frac{\partial^{2}}{\partial r^{2}}-j_{n}^{2}\right) H_{1 c}+C_{55} \varepsilon_{11} e_{15}^{-2}\left(\frac{\partial^{2}}{\partial r^{2}}+\frac{1}{r} \frac{\partial}{\partial r}-j_{n}^{2}\right) H_{2 c} \\
p_{0 c}(r, n)=\nu_{0 c}(r, n)-\left.H_{1 c}\right|_{t=0}, \quad \dot{p}_{0 c}(r, n)=\dot{\nu}_{0 c}(r, n)-\left.\dot{H}_{1 c}\right|_{t=0}
\end{gathered}
$$

В равенствах (11) и ниже штрих обозначает дифференцирование по переменной $r$. ний

Функции $f_{1}(r), f_{2}(r), f_{3}(r)$ определяются из дифференциальных уравне-

$$
f_{1}^{\prime \prime \prime}(r)=0, \quad f_{2}^{\prime \prime}(r)=0, \quad f_{3}^{\prime \prime \prime}(r)=0 .
$$

Начально-краевая задача (12)-(14) относительно $p_{c}(r, n, t), \chi_{c}(r, n, t)$ peшается при помощи структурного алгоритма метода конечных интегральных преобразований (КИП) [6]. Введём на сегменте $[k, 1]$ вырожденное КИП с неизвестными компонентами $K_{1}\left(\lambda_{i n}, r\right), K_{2}\left(\lambda_{i n}, r\right)$ вектор-функции ядра преобразования: 


$$
\begin{aligned}
& G\left(\lambda_{i n}, n, t\right)=\int_{k}^{1} p_{c}(r, n, t) K_{1}\left(\lambda_{i n}, r\right) r d r, \\
& p_{c}(r, n, t)=\sum_{i=1}^{\infty} G\left(\lambda_{i n}, n, t\right) K_{1}\left(\lambda_{i n}, r\right)\left\|K_{i n}\right\|^{-2}, \\
& \chi_{c}(r, n, t)=\sum_{i=1}^{\infty} G\left(\lambda_{i n}, n, t\right) K_{2}\left(\lambda_{i n}, r\right)\left\|K_{i n}\right\|^{-2}, \\
& \left\|K_{i n}\right\|^{2}=\int_{k}^{1} K_{1}^{2}\left(\lambda_{i n}, r\right) r d r,
\end{aligned}
$$

где $\lambda_{i n}, i \in \mathbb{N}$ - положительные параметры, образующие счётное множество; $\left\|K_{i n}\right\|$ - норма вектор-функции вырожденного преобразования. При этом круговые частоты осесимметричных колебаний цилиндра $\omega_{i n}$ связаны с $\lambda_{i n}$ зависимостью

$$
\omega_{i n}=\frac{\lambda_{i n}}{b} \sqrt{\frac{C_{55}}{\rho}} .
$$

Подвергая систему уравнений (12) преобразованиям в соответствии со структурным алгоритмом [6], получаем счётное множество задач Коши для трансформанты $G\left(\lambda_{i n}, n, t\right)$ :

$$
\begin{gathered}
\ddot{G}\left(\lambda_{i n}, n, t\right)+\lambda_{i n}^{2} G\left(\lambda_{i n}, n, t\right)=-F\left(\lambda_{i n}, n, t\right), \\
n=0,1,2, \ldots, i=1,2,3, \ldots, \\
G\left(\lambda_{i n}, n, 0\right)=G_{0}\left(\lambda_{i n}, n\right), \quad \dot{G}\left(\lambda_{i n}, n, 0\right)=\dot{G}_{0}\left(\lambda_{i n}, n\right)
\end{gathered}
$$

и однородную краевую задачу для компонент $K_{1}, K_{2}$ ядра КИП:

$$
\begin{aligned}
& \left(\frac{d^{2}}{d r^{2}}+\frac{1}{r} \frac{d}{d r}-\frac{1}{r^{2}}-j_{n}^{2}+\lambda_{i n}^{2}\right) K_{1}+\left(\frac{d^{2}}{d r^{2}}+\frac{2}{r} \frac{d}{d r}-j_{n}^{2}\right) K_{2}=0 \\
& \left(\frac{d^{2}}{d r^{2}}-j_{n}^{2}\right) K_{1}-C_{55} \varepsilon_{11} e_{15}^{-2}\left(\frac{d^{2}}{d r^{2}}+\frac{1}{r} \frac{d}{d r}-j_{n}^{2}\right) K_{2}=0 \\
& r=1, k: \quad K_{1}\left(\lambda_{i n}, k\right)=0, \quad K_{2}\left(\lambda_{i n}, k\right)=0 \\
& \text { а) } \quad\left[\frac{d K_{1}}{d r}-K_{1}\right]_{r=1}=0,\left.\quad \frac{d K_{2}}{d r}\right|_{r=1}=0 ; \\
& \text { б) }\left[\frac{d K_{1}}{d r}-K_{1}+\frac{d K_{2}}{d r}\right]_{r=1}=0, \quad K_{2}\left(\lambda_{i n}, 1\right)=0
\end{aligned}
$$

Здесь

$$
\begin{gathered}
F\left(\lambda_{i n}, n, t\right)=\int_{k}^{1}\left[B_{1} K_{1}+B_{2} K_{2}\right] r d r, \quad G_{0}\left(\lambda_{i n}, n\right)=\int_{k}^{1} p_{0 c} K_{1} r d r \\
\dot{G}_{0}\left(\lambda_{i n}, n\right)=\int_{k}^{1} \dot{p}_{0 c} K_{1} r d r .
\end{gathered}
$$

С учётом (18) решение уравнения (17) записывается в виде 


$$
\begin{aligned}
G\left(\lambda_{i n}, n, t\right)=G_{0} \cos \lambda_{i n} t+\dot{G}_{0} \lambda_{i n}^{-1} & \sin \lambda_{i n} t+ \\
& +\lambda_{i n}^{-1} \int_{0}^{t} F\left(\lambda_{i n}, n, t\right) \sin \lambda_{i n}(t-\tau) d \tau .
\end{aligned}
$$

Система (19) сводится к следующему разрешающему дифференциальному уравнению относительно $K_{1}\left(\lambda_{i n}, r\right)$ :

$$
K_{1}^{I V}+\frac{4}{r} K_{1}^{\prime \prime \prime}+b_{1} K_{1}^{\prime \prime}+\frac{b_{2}}{r} K_{1}^{\prime}+j_{n}^{2}\left(b_{3}+\frac{b_{4}}{r^{2}}\right) K_{1}=0,
$$

где $b_{1}=\lambda_{i n}^{2} b_{4}-2 j_{n}^{2}, b_{2}=3 b_{4}-4 j_{n}^{2}, b_{3}=j_{n}^{2}-\lambda_{i n}^{2} b_{4}, b_{4}=\left(C_{55} \varepsilon_{11}\right)\left(C_{55} \varepsilon_{11}+e_{15}^{2}\right)^{-1}$.

Частное решение дифференциального уравнения (22) находится методом разложения функции $K_{1}$ в степенной ряд:

$$
K_{1}=r^{\beta} \sum_{f=0,2,4}^{\infty} a_{f} r^{f}, \quad \beta=\text { const. }
$$

После подстановки (23) в (22), приравнивая нулю все множители с одинаковой степенью, получаем систему алгебраических уравнений относительно $a_{f}$, решение которых позволяет определить все коэффициенты относительно $a_{0}$. Полагая $a_{0}=\beta(\beta+1)$, получаем выражения для определения $a_{f}$ :

$$
\begin{aligned}
& a_{2}=-\frac{b_{1} \beta(\beta-1)+b_{2} \beta+j_{n}^{2} b_{4}}{(\beta+2)(\beta+3)}, \\
& a_{f}=-\frac{j_{n}^{2} b_{3} a_{f-4}+\left[b_{1}(\beta+f-2)(\beta+f-3)+b_{2}(\beta+f-2)+j_{n}^{2} b_{4}\right] a_{f-2}}{(\beta+f+1)(\beta+f)(\beta+f-1)(\beta+f-2)}, \\
& f=4,6,8 \ldots,
\end{aligned}
$$

а также характеристическое уравнение для определения параметра $\beta$ :

$$
\beta(\beta+1)(\beta-1)(\beta-2)=0 .
$$

При $\beta=0$ и $\beta=1$ получаем первые два линейно независимых решения. Оставшиеся решения будем искать в следующем виде:

$$
K_{1}=\ln (r) \sum_{f=0,2,4}^{\infty} a_{f} r^{f+\beta}+\sum_{f=0,2,4}^{\infty} m_{f} r^{f-\beta}
$$

где $\beta=0$ и $\beta=1$.

Принимая во внимание (24) и приравнивая все множители разложения (22) с одинаковой степенью, определяем коэффициенты $m_{f}$ :

$$
\begin{gathered}
m_{0}=-\frac{6 a_{2}}{j_{n}^{2} b_{4}} \quad \text { при } \beta=0, \quad m_{0}=\frac{2 a_{0}}{2 b_{1}-b_{2}+j_{n}^{2} b_{4}} \quad \text { при } \beta=1, \\
m_{2}=1,
\end{gathered}
$$




$$
\begin{aligned}
& m_{f}=-\left\{\left[b_{1}(2 f-2 \beta-5)+b_{2}\right] a_{f-4}+\right. \\
& +\left[4 f^{3}+6(1-4 \beta) f^{2}+(24 \beta-2) f+2-8 \beta\right] a_{f-2}+ \\
& \left.+j_{n}^{2} b_{3} m_{f-4}+\left[b_{1}(f-\beta-2)(f-\beta-3)+b_{2}(f-\beta-2)+j_{n}^{2} b_{4}\right] m_{f-2}\right\} \times \\
& \quad \times[f(f-1)(f-2)(f-4 \beta+1)]^{-1} \quad f=4,6,8, \ldots
\end{aligned}
$$

В результате общее решение дифференциального уравнения (22) имеет вид

$$
K_{1}\left(\lambda_{i n}, r\right)=\sum_{w=1}^{4} D_{w(i n)} N_{w}\left(\lambda_{i n}, r\right)
$$

где

$$
\begin{gathered}
N_{1}\left(\lambda_{i n}, r\right)=\sum_{f=0,2,4, \ldots} a_{f} r^{f}, \quad N_{2}\left(\lambda_{i n}, r\right)=\sum_{f=0,2,4, \ldots} a_{f} r^{f+1}, \\
N_{3}\left(\lambda_{i n}, r\right)=\ln (r) N_{1}\left(\lambda_{i n}, r\right)+\sum_{f=0,2,4, \ldots} m_{f} r^{f}, \\
N_{4}\left(\lambda_{i n}, r\right)=\ln (r) N_{2}\left(\lambda_{i n}, r\right)+\sum_{f=0,2,4, \ldots} m_{f} r^{f-1} .
\end{gathered}
$$

Используя зависимости между $K_{1}\left(\lambda_{i n}, r\right)$ и $K_{2}\left(\lambda_{i n}, r\right)$, полученные в процессе приведения (19) к (22), получаем выражения для второй компоненты ядра преобразований:

$$
\begin{aligned}
& K_{2}\left(\lambda_{i n}, r\right)=-\left(b_{4} j_{n}^{2}\right)^{-1} \sum_{w=1}^{4} D_{w(i n)} P_{w}\left(\lambda_{i n}, r\right), \\
& P_{w}\left(\lambda_{i n}, r\right)=\left[r \frac{d^{3}}{d r^{3}}+3 \frac{d^{2}}{d r^{2}}+\left(\lambda_{i n}^{2} b_{4}-j_{n}^{2}\right) r \frac{d}{d r}+\right. \\
& \left.+b_{4}\left(2 \lambda_{i n}^{2}-j_{n}^{2} \frac{e_{15}^{2}}{C_{55} \varepsilon_{11}}\right)-2 j_{n}^{2}\right] N_{w}\left(\lambda_{i n}, r\right) .
\end{aligned}
$$

Подстановка (25), (26) в граничные условия (20) формирует однородную систему уравнений относительно постоянных $D_{1 i n}, D_{2 i n}, D_{3 i n}, D_{4 i n}$. Разыскивая её нетривиальные решения, получаем трансцендентное уравнение для вычисления собственных значений $\lambda_{i n}$ :

$$
\operatorname{det}\left[B_{s w}\right]=0, \quad s, w=1,2,3,4
$$

$$
B_{1 w}=N_{w}\left(\lambda_{i n}, k\right), \quad B_{2 w}=P_{w}\left(\lambda_{i n}, k\right)
$$

a) $B_{3 w}=N_{w}^{\prime}\left(\lambda_{i n}, 1\right)-N_{w}\left(\lambda_{i n}, 1\right), \quad B_{4 w}=P_{w}^{\prime}\left(\lambda_{i n}, 1\right)$;

б) $B_{3 w}=N_{w}^{\prime}\left(\lambda_{i n}, 1\right)-N_{w}\left(\lambda_{i n}, 1\right)+P_{w}^{\prime}\left(\lambda_{i n}, 1\right), \quad B_{4 w}=P_{w}\left(\lambda_{i n}, 1\right)$.

Без ограничения общности принимая $D_{1 \text { in }}=1$, оставшиеся постоянные интегрирования определяются при решении системы неоднородных уравнений

$$
\left[\begin{array}{lll}
B_{22} & B_{23} & B_{24} \\
B_{32} & B_{33} & B_{34} \\
B_{42} & B_{43} & B_{44}
\end{array}\right]\left[\begin{array}{l}
D_{2 i n} \\
D_{3 i n} \\
D_{4 i n}
\end{array}\right]=-\left[\begin{array}{l}
B_{21} \\
B_{31} \\
B_{41}
\end{array}\right],
$$

записанной в матричном виде. 
3. Расчётные соотношения. Заключительным этапом исследования является определение функций $f_{1}(r), f_{2}(r), f_{3}(r)$, входящих в представления (10). Для этой цели воспользуемся дифференциальными уравнениями (15) и соответствующими граничными условиями (11). В результате имеем
a) $f_{1}(r)=b_{4}\left[r^{2}-(k+1) r+k\right](1-k)^{-1}$ $f_{2}(r)=\frac{e_{15}^{2}}{e_{15}^{2}+C_{55} \varepsilon_{11}}(r-k) ;$
б) $f_{3}(r)=(k-1)^{-2}\left[-r^{2}+2 r+k(k-2)\right]$.

Применяя к трансформанте (21) последовательно формулы обращения КИП (16), а затем конечных косинус-преобразований Фурье, с учётом (10) получаем следующие разложения для $\nu(r, z, t), \phi(r, z, t)$ :

$$
\begin{aligned}
\nu(r, z, t) & =\sum_{n=0}^{\infty} \Omega^{-1}\left[H_{1}(r, n, t)+\sum_{i=1}^{\infty} G\left(\lambda_{i n}, n, t\right) K_{1}\left(\lambda_{i n}, r\right)\left\|K_{i n}\right\|^{-2}\right] \cos j_{n} z, \\
\phi(r, z, t) & =\sum_{n=0}^{\infty} \Omega^{-1}\left[H_{2}(r, n, t)+\sum_{i=1}^{\infty} G\left(\lambda_{i n}, n, t\right) K_{2}\left(\lambda_{i n}, r\right)\left\|K_{i n}\right\|^{-2}\right] \cos j_{n} z,
\end{aligned}
$$

где $\Omega_{n}=\left\{\begin{array}{cc}L, & n=0, \\ L / 2, & n \neq 0 .\end{array}\right.$

Разность потенциалов $Q(t)$ в задаче прямого пьезоэффекта определяется следующим образом [7]:

$$
Q(t)=\left(z_{2}-z_{1}\right)^{-1} \int_{z_{1}}^{z_{2}} \phi(1, z, t) d z,
$$

где $z_{1}, z_{2}$ - соответственно нижняя и верхняя границы внешней цилиндрической поверхности, на которую действует нагрузка $q(z, t)$.

4. Численный анализ результатов. В качестве примера рассматривается пьезокерамический цилиндр из состава ЦТС-19 [2] при действии на внешней радиальной поверхности следующего электрического воздействия:

$$
V(z, t)=V\left[H\left(z-z_{1}\right)-H\left(z-z_{2}\right)\right] \sin \theta t
$$

где $V$ - амплитудное значение нагрузки, $\theta$ - частота вынужденных колебаний, $H(z)$ - единичная функция Хэвисайда.

В таблице приведены собственные значения $\lambda_{i n}$ свободных осесимметричных колебаний элемента для различных значений относительной толщины

Собственные значения $\lambda_{i n}$ свободных осесимметричных колебаний пьезокерамического цилиндра

\begin{tabular}{c|c|c|c|c|c|c|c|c}
\hline \multirow{2}{*}{} & \multicolumn{3}{|c|}{ краевые условия «а» } & \multicolumn{3}{c}{ краевые условия «б» } \\
\cline { 2 - 9 } & \multicolumn{2}{|c|}{$b / a=2$} & \multicolumn{2}{|c|}{$b / a=5$} & \multicolumn{2}{c}{$b / a=2$} & \multicolumn{2}{c}{$b / a=5$} \\
\cline { 2 - 9 } & $i=1$ & $i=2$ & $i=1$ & $i=2$ & $i=1$ & $i=2$ & $i=1$ & $i=2$ \\
\hline$n=0$ & 2,50 & 11,63 & 0,75 & 7,41 & 2,05 & 11,50 & 0,63 & 7,09 \\
$n=1$ & 4,68 & 12,27 & 3,99 & 8,30 & 3,91 & 11,93 & 3,40 & 8,02 \\
$n=3$ & 8,32 & 14,07 & 7,95 & 10,78 & 7,16 & 13,58 & 6,98 & 10,43 \\
\hline
\end{tabular}


$b / a(h=b)$. При увеличении параметра $b / a$ жёсткость конструкции понижается и соответственно наблюдается уменьшение $\lambda_{i n}$. Кроме того, подтверждаются известные экспериментальные и теоретические $[1,2]$ результаты, что электрические краевые условия оказывают влияние на частоту собственных колебаний, и более низкие значения наблюдаются при подключении электродированных поверхностей к источнику электрического напряжения (краевые условия «б»).

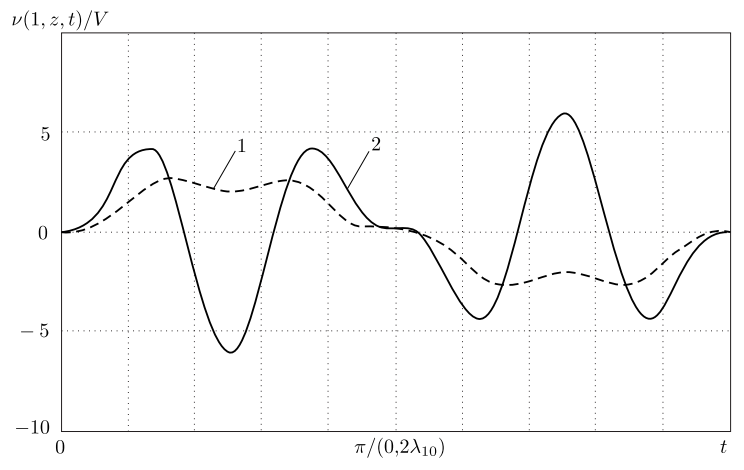

Рис. 1. Зависимость тангенциальных перемещений внешней криволинейной поверхности цилиндра от времени

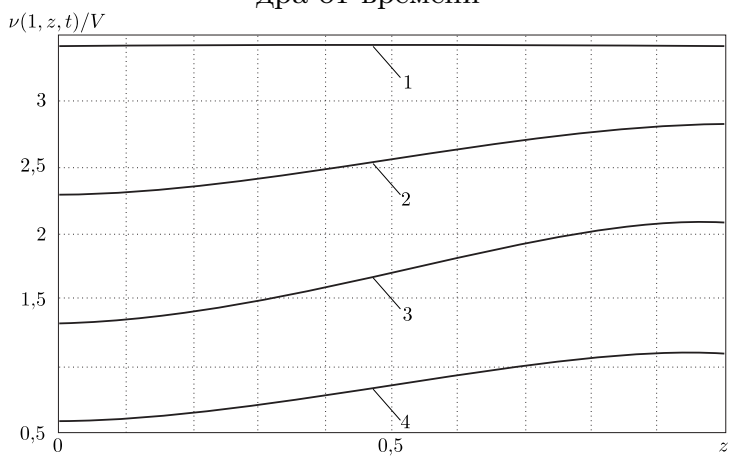

Рис. 2. Изменение амплитудных значений тангенциальных перемещений по высоте цилиндра при различных загружениях
На рис. 1 показаны графики изменения по времени $t$ тангенциальных перемещений внешней криволинейной поверхности $\nu(1, z, t)$. Цифрами обозначены кривые, соответствующие следующим вариантам: 1) $\theta=0,6 \lambda_{10}$, 2) $\theta=0,2 \lambda_{10}\left(\lambda_{10}-\right.$ собственные значения основного тона колебаний, $b / a=5, z_{1}=0, z_{2}=$ $=L)$. Результаты расчёта показывают, что даже при действии гармонической нагрузки допущение об установившемся режиме вынужденных колебаний $[1,2]$ можно использовать только тогда, когда частотные характеристики внешнего воздействия $\theta$ существенно меньше собственного значения $\lambda_{10}$ цилиндра.

На рис. 2 изображены амплитудные значения тангенциальных перемещений внешней радиальной поверхности цилиндра при различной степени его загружения. Цифры соответствуют следующим случаям: 1) $z_{1}=$ $=0, z_{2}=L$; 2) $z_{1}=0, z_{2}=3 L / 4$; 3) $z_{1}=0, z_{2}=L / 2$;4) $z_{1}=0$, $z_{2}=L / 4$. Следует подчеркнуть, что область внешнего воздействия оказывает существенное влияния как в количественном, так и в качественном отношениях на деформированное состояние системы.

В заключение можно отметить, что рассмотренный метод решения позволяет также получить результаты для случая «короткого замыкания» [2], используемые при определении электроакустической чувствительности пьезопреобразователей, работающих в резонансном режиме.

\section{БИБЛИОГРАФИЧЕСКИЙ СПИСОК}

1. Партон В.З., Кудрявцев Б. А. Электромагнитоупругость пьезоэлектрических и электропроводных тел. М.: Наука, 1988. 470 c. [Parton V.Z., Kudriavcev B.A. Electromagnetoelasticity of Piezoelectric and Electroconductive Bodies. Moscow: Nauka, 1988. 470 pp.] 
2. Гринченко В.Т., Улитко А.Ф., Шулъга Н.А. Механика связанных полей в элементах конструкций. Т. 5: Электроупругость. Киев: Наук. думка, 1989. 279 с. [Grinchenko V. T., Ulitko A.F., Shul'ga N. A. Mechanics of Coupled Fields in Structural Elements. Vol. 5: Electroelasticity. Kiev: Nauk. Dumka, 1989. 279 pp.]

3. Шулъга Н. А., Болкисев А. М. Колебания пьезоэлектрических тел. Киев: Наук. думка, 1990. 228 c. [Shul'ga N. A., Bolkisev A. M. Vibrations of Piezoelectric Bodies. Kiev: Nauk. Dumka, 1990. 2228 pp.]

4. Сеницкий Ю.Э. Динамическая задача электроупругости для неоднородного цилиндра // ПММ, 1993. Т. 57, №1. С. 116-122; англ. пер.: Senitskii Yu. E. The dynamic problem of electroelasticity for a non-homogeneous cylinder // J. Appl. Math. Mech., 1993. Vol.57, no. 1. Pp. 133-139.

5. Ермолов И.Н. Ультразвуковые преобразователи для неразрушающего контроля. М.: Машиностроение, 1986. 280 с. [Ermolov I. N. Ultrasonic transducers for nondestructive testing. Moscow: Mashinostroenie, 1986. 280 pp.]

6. Сеницкий Ю.Э. Многокомпонентное обобщенное конечное интегральное преобразование и его приложение к нестационарным задачам механики // Изв. вузов. Матем., 1991. №4. C. 57-63; англ. пер.: Senitskii Yu. E. A multicomponent generalized finite integral transformation and its application to nonstationary problems in mechanics // Soviet Math. (Iz. VUZ), 1991. Vol. 35, no. 4. Pp. 55-61.

7. Тамм И. Е. Основы теории электричества. М.: Наука, 1989. 504 с. [Tamm I. E. Basic Theory of Electricity. Moscow: Nauka, 1989. 504 pp.]

Поступила в редакцию 28/XII/2010;

в окончательном варианте $-29 / \mathrm{IV} / 2011$.

MSC: 74B05; 74F15, 74S20

\section{FORCED OSCILLATION OF PIEZOCERAMIC CYLINDER WITH CIRCUMPOLARIZATION OF MATERIAL}

\section{A. Shlyakhin}

Samara State University of Architecture and Civil Engineering,

194, Molodogvardeyskaya st., Samara, 443001, Russia.

E-mail: d-612-mit2009@yandex.ru

The nonstationary elasto-electrodynamics problem for an anisotropic piezoceramic cylinder of finite size with circumpolarization of material is considered for the case when the tangential stresses and the electric potential influence on the radial outside of the cylinder as the arbitrary functions of axial coordinate and time. The new closed solution is obtained by the method of expansion in vector eigenfunctions in the form of structural algorithm of finite transformations. The constructed algorithm allows to determine the eigenfrequencies, stress-strain state of the element, and all the components of the induced electric field.

Key words: coupled problem of electric elasticity, cylinder of final sizes, axisymmetric dynamic load.

Original article submitted 28/XII/2010; revision submitted $29 / \mathrm{IV} / 2011$.

Dmitriy A. Shlyakhin (Ph. D. (Techn.)), Associate Professor, Dept. of Resistance of Materials \& Construction Mechanics. 